\title{
Outcomes in elderly patients admitted to the intensive care unit with solid tumors
}

\author{
Edouard Auclin 1,2,3,4*, Anaïs Charles-Nelson ${ }^{5}$, Baptiste Abbar², Emmanuel Guérot², Stéphane Oudard,4, \\ Caroline Hauw-Berlemont ${ }^{2,4}$, Constance Thibault 3,4, Alexandra Monnier ${ }^{2,4}$, Jean-Luc Diehl ${ }^{2,4}$, \\ Sandrine Katsahian ${ }^{4,5}$, Jean-Yves Fagon ${ }^{2,4}$, Julien Taieb ${ }^{1,4}$ and Nadia Aissaoui ${ }^{2,4}$
}

\begin{abstract}
Background: As the population ages and cancer therapies improve, there is an increased call for elderly cancer patients to be admitted to the intensive care unit (ICU). This study aimed to assess short-term survival and prognostic factors in critically ill patients with solid tumors aged $\geq 65$ years.

Methods: We conducted a retrospective study. The primary endpoint was ICU mortality. Resumption of anticancer therapy in patients who survived the ICU stay and 90-day mortality were secondary endpoints. All patients aged $\geq 65$ years admitted to the ICU of Georges Pompidou Hospital (Paris, France) between 2009 and 2014 were eligible.

Results: Of 2327 eligible elderly patients (EP), 262 (75.0 \pm 6.7 years) with solid tumors were analyzed. These patients were extremely critically ill (SAPS $261.9 \pm 22.5$ ), and $60.3 \%$ had metastatic disease. Gastrointestinal, lung and genitourinary cancers were the most common types of tumors. Mechanical ventilation was required in $51.5 \%$ of patients, inotropes in $48.1 \%$ and dialysis in $12.6 \%$. Most patients (66.7\%) were admitted for reasons unrelated to cancer, including sepsis (30.5\%), acute respiratory failure (28.2\%) and neurological problems (8.0\%). ICU mortality in patients with cancer was 33.6 versus $32.6 \%$ among patients without cancer $(p=0.75)$. Among the cancer $E P$, the 90 -day mortality was $51.9 \%(n=136)$. In multivariate analysis, increased SAPS 2 score and primary tumor site were associated with 90-day death, whereas previous anticancer therapies and poor performance status were not. Among survivor patients from ICU with anti-tumoral treatment indication, 77 (52.7\%) had resumption of anticancer treatment.

Conclusions: Elderly solid tumor patients admitted to the ICU had a mortality rate similar to EP without cancer. Prognostic factors for 90 -day mortality were more related to severity of clinical status at admission than the presence or stage of cancer, suggesting that early admission of EP with cancer to the ICU is appropriate.
\end{abstract}

Keywords: Elderly, Cancer, Outcome, Intensive care, Survival, Treatment

\section{Background}

Cancer is the leading cause of mortality in France, ahead of cardiovascular diseases. In 2015 there were 385,000 new cases of cancer in France, with 149,500 cancerrelated deaths [1]. The median age at diagnosis of cancer is 65 years, and the rate of cancer diagnosis increases with age in both males and females [2].

${ }^{*}$ Correspondence: ed.auclin@gmail.com

${ }^{4}$ Université Paris Descartes, Paris, France

Full list of author information is available at the end of the article

Remarkable advances have been made in the early diagnosis and management of patients with malignancies, resulting in dramatic improvements in overall survival rates $[3,4]$. As a result, increasing numbers of oncology patients are admitted to the intensive care unit (ICU), for either life-threatening cancer-related complications, treatment-associated side effects or standard critical care admission indications [5].

Overall, survival rates in critically ill patients with active cancer appear to be increasing [5]. However, studies conducted among patients with hematological malignancies or lung cancer indicate that ICU mortality 
in these patients is high [6-8]. In addition, a number of unanswered questions remain, including a lack of followup data on patients who survive a stay in ICU, and data on the clinical course and anticancer treatment continuation rates after the critical illness are very limited $[8,9]$. Furthermore, there is a lack of data on ICU patients with non-hematological malignancies.

The aim of this study was to assess patient outcomes and identify factors associated with 90-day mortality and antineoplastic treatment resumption in elderly patients with metastatic or non-metastatic solid cancers admitted to the ICU.

\section{Methods}

\section{Study design}

This retrospective observational study was conducted between 2009 and 2014 in the ICU of the European Georges Pompidou Hospital (Paris, France). The protocol was approved by the local institutional ethics committee, and the study was performed in accordance with the ethical standards laid down in the 1964 Declaration of Helsinki.

\section{Patients}

Consecutive patients were eligible for inclusion if they were aged 65 years or older, with or without a diagnosis of malignant solid tumor. Patients with hematological malignancy, an ICU stay of $<24 \mathrm{~h}$ with limitation of active therapies, cancer diagnosed during the ICU stay, or cancer remission of more than 5 years were excluded.

\section{Data collection}

Data were collected retrospectively from patients' computerized medical files using the Dxcare software. Demographic data, performance status, cancer data (primary tumor, presence of metastases, metastatic sites, number of previous anticancer treatments, type of treatment and neutropenia status), reasons for ICU admission, status at admission assessed using the SAPS 2 score [10], life-support therapies used and the duration of use (mechanical ventilation, inotropes), and biological data at admission were collected. Reasons for admission were classified in two ways: one based on relationship with cancer (related to cancer progression or antineoplastic treatment toxicity, or no link with cancer) and the second based on medical diagnosis at admission (sepsis, acute heart failure, hemorrhagic shock, hypovolemia, acute respiratory failure, chronic bronchitis exacerbation, neurological reasons (i.e., coma and/or seizure), acute kidney failure, electrolytic disorders, cardiorespiratory shock, post-surgery, medical surveillance, unknown shock). The reason for admission was considered as the symptom at the origin of the ICU request.

\section{Outcomes}

ICU mortality rates were determined for elderly patients with and without solid tumors. For those with solid tumors, in-hospital mortality, 90-day mortality and anticancer treatment resumption were also assessed.

\section{Statistical analysis}

Statistical analysis was performed using R software. For quantitative variables, mean and standard deviations were calculated. Discrete variables are presented as percentages. Comparisons between patient characteristics were performed using Chi-square or Fisher's exact test for discrete variables and using the unpaired $t$ test, Wilcoxon sign-rank test or analysis of variance for continuous variables.

Predictive factors for 90-day mortality were tested in univariate and multivariate analyses using a Cox proportional hazards model. The proportional hazards assumption was verified using the Schoenfeld residuals method. Variables included in the final multivariable models were selected according to their physiological relevance and statistical significance in univariate analysis, using a $p$ value threshold of 0.10 . Two multivariable analyses were conducted among cancer patients: the first included only variables available on admission: age, comorbidity, performance status, cancer type, metastatic status, SAPS 2 and biological data. The second model added inICU management variables (respiratory, circulatory or renal support techniques). Analyses were repeated using forward stepwise analysis to assess the consistency of results. Collinearity was assessed by calculating variance inflation factors. In addition, logistic regression was used to assess factors predictive of definitive anticancer treatment cessation, and the final model was defined using a stepwise method. For all analyses, a $p$ value $<0.05$ was considered statistically significant.

\section{Results}

A total of 4185 patients were admitted to the ICU over the study period. Of these, 2327 were classified as elderly (age $\geq 65$ years), 332 (14.3\%) of whom had a solid tumor, and $262(11.3 \%)$ were included in the final analysis (Fig. 1).

\section{Elderly patients with or without solid tumor}

The mean age \pm standard deviation of all elderly patients $(n=2327)$ was $77.1 \pm 8.1$ years (1311 male; $56.3 \%)$. Patients with cancer were significantly younger than those without cancer $(75.2 \pm 6.7$ vs $79.0 \pm 8.2$ years; $p<0.0001)$. The mean \pm standard deviation IGS2 score in all elderly patients was $59.0 \pm 23.0$. The SAPS 2 score on admission was significantly higher in cancer versus noncancer patients $(61.9 \pm 22.5$ vs $56.9 \pm 22.4 ; p<0.0001)$, as 


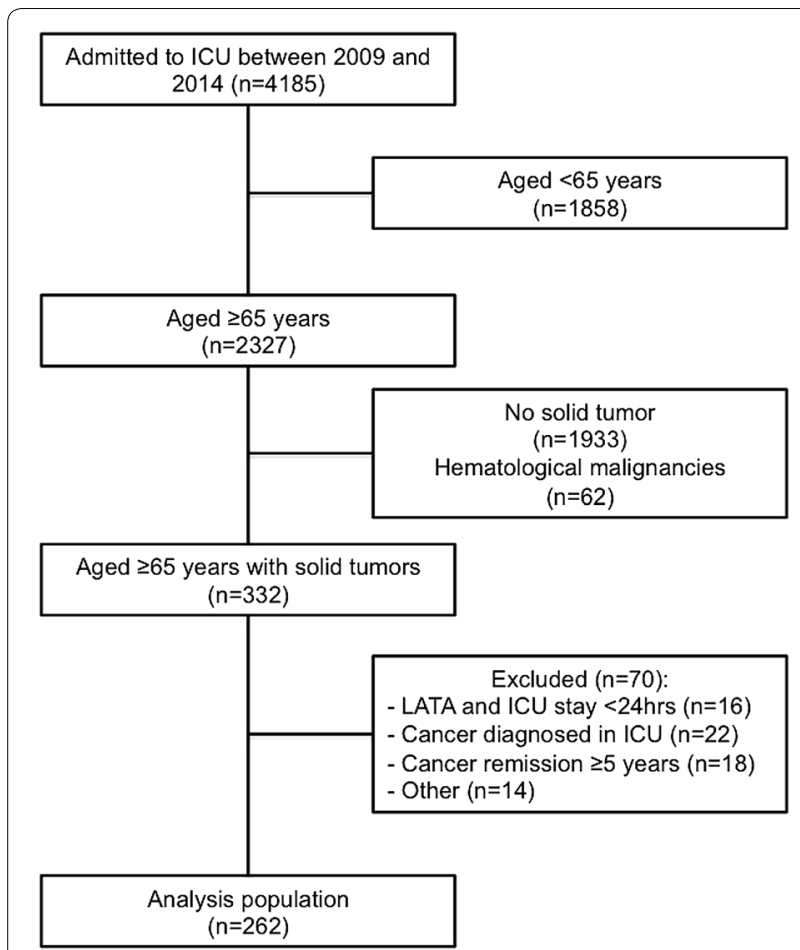

Fig. 1 Flow of patients through the study. ICU intensive care unit, LATA limitation of active therapies

was the McCabe score $(2.1 \pm 0.6$ vs $1.3 \pm 0.6 ; p<0.025)$. Further details of patient characteristics within the overall population are shown in Additional file 1: Table S1.

The ICU mortality for all elderly patients was $32.5 \%$ and did not differ between those with and without solid tumor (33.6 vs $32.7 \%$; $p=0.78$ ). Cancer was not associated with in-ICU survival (OR for patients without cancer 0.96 ; 95\% CI $0.73-1.26$; $p=0.78$ ).

\section{Elderly patients with solid tumor Characteristics}

Full details of demographic and clinical characteristics for patients with solid tumor are reported in Table 1, overall and based on survivorship. Gastrointestinal, lung and genitourinary cancers were the most common types of tumors (Table 1). Sixty-three percent of patients had received at least one previous line of systemic antitumoral treatment (Table 1). Sepsis and acute respiratory failure were the two most common reasons for ICU admission (Table 2). Based on SAPS 2 score, patients were critically ill at admission and 135 (51.5\%) required mechanical ventilation (Table 2). Median ICU stay was 4.0 days (interquartile range 2.0-7.0). Laboratory parameters for the 262 elderly patients with solid tumors are shown in Additional file 1: Table S2.

\section{Outcomes}

The ICU mortality rate in elderly solid tumor patients was $33.6 \%(n=88)$, the in-hospital mortality rate was $43.9 \%$ $(n=115)$, while the 90 -day mortality was $51.9 \%(n=136$, lost to follow-up $n=14$ ). Out of the 174 patients with solid tumor who survived the ICU stay, 28 did not resume anticancer therapy because there was no treatment indication (i.e., localized tumor). Out of the 146 patients with advanced disease who theoretically had an indication for additional cancer therapy, $77(52.7 \%)$ received treatment, $54(37.0 \%)$ did not receive treatment, and 15 patients (10.3\%) were lost to follow-up. Characteristics of the 146 ICU survivors with anti-tumoral treatment indication are presented in Additional file 1: Table S3.

\section{Prognostic factors analysis Univariate analysis}

Variables significantly associated with 90-day death were $\operatorname{sex}(p=0.03)$, SAPS 2 score $(p<0.0001)$, mechanical ventilation $(p<0.0001)$, inotrope use $(p<0.0001)$, limitation of active therapies during ICU stay $(p<0.0001)$, primary tumor site $(p=0.005)$, leukocyte count $(p<0.0001)$, blood $\mathrm{pH}(p<0.0001)$, lactate levels $(p<0.0001)$, aspartate aminotransferase level $(p<0.0001)$, alanine aminotransferase level $(p=0.01)$, bilirubin $(p=0.04)$ and albumin $(p=0.02)$ (Table 3). Performance status was not associated with 90 -day mortality in univariate analysis $(p=0.07)$, as was metastatic status $(p=0.82)$.

\section{Multivariate analysis}

High SAPS 2 score (HR 1.05; 95\% CI 1.03-1.06, $p<0.0001)$ and primary tumor site $(p=0.01)$ were significantly associated with 90-day death in patients with solid tumors admitted in ICU. When artificial life-support techniques were added to the model, SAPS 2, primary tumor site, metastatic status and lactates were predictive of 90-day death (Additional file 1: Table S4). The number and type of previous anticancer therapies and the performance status were not associated with death in multivariate analysis.

For the 146 patients with an indication for ongoing anticancer therapy, those with a gastrointestinal $(p=0.01)$ or lung $(p=0.02)$ tumor, or who had a performance status of 3 or $4(p=0.001)$ or who were admitted in ICU because of cancer progression $(p=0.04)$, were significantly less likely to have antineoplastic treatment restarted after ICU discharge (Table 4).

\section{Discussion}

This study provides interesting data about outcomes in elderly patients with active solid tumors admitted to the ICU, who comprised $14.3 \%$ of all elderly patients with an 
Table 1 Baseline patient demographics and clinical characteristics for the overall study population and by survivor status

\begin{tabular}{|c|c|c|c|c|}
\hline & Overall $(n=262)$ & ICU survivors $(n=174)$ & ICU non-survivors $(n=88)$ & $p$ value \\
\hline Age (years) & $75.2 \pm 6.7$ & $75.7 \pm 6.8$ & $74.3 \pm 6.5$ & 0.13 \\
\hline Male & $162(61.8)$ & $101(58.0)$ & $61(69.3)$ & 0.08 \\
\hline \multicolumn{5}{|l|}{ Comorbidities } \\
\hline Tobacco use & $106(40.5)$ & $65(37.4)$ & $41(46.6)$ & 0.15 \\
\hline Diabetes & $57(21.8)$ & $35(20.1)$ & $22(25)$ & 0.36 \\
\hline Chronic respiratory failure & $33(12.6)$ & $23(13.2)$ & $10(11.4)$ & 0.18 \\
\hline Chronic kidney failure & $29(11.1)$ & $23(13.2)$ & $6(6.8)$ & 0.12 \\
\hline Primary tumor site & & & & 0.02 \\
\hline Gastrointestinal & $71(27.1)$ & $52(29.9)$ & $19(21.6)$ & \\
\hline Lung & $68(26)$ & $45(25.9)$ & $23(26.1)$ & \\
\hline Genitourinary & $60(22.9)$ & $43(24.7)$ & $17(19.3)$ & \\
\hline Head and neck & $31(11.8)$ & $11(6.3)$ & $20(22.7)$ & \\
\hline Breast & $18(6.9)$ & $13(7.5)$ & $5(5.7)$ & \\
\hline Gynecological & $9(3.4)$ & $7(4)$ & $2(2.3)$ & \\
\hline Sarcoma & $3(1.1)$ & $2(1.2)$ & $1(1.1)$ & \\
\hline Unknown & $2(0.8)$ & $1(0.6)$ & $1(1.1)$ & \\
\hline \multicolumn{5}{|l|}{ Metastasis } \\
\hline Yes & $158(60.3)$ & $110(63.2)$ & $48(54.6)$ & 0.17 \\
\hline Other & $66(25.3)$ & $42(24.3)$ & $24(27.3)$ & 0.58 \\
\hline Lymph node & $58(22.2)$ & $39(22.5)$ & $19(21.6)$ & 0.93 \\
\hline Lung & $57(21.8)$ & $41(23.7)$ & $16(18.2)$ & 0.32 \\
\hline Bone & $46(17.6)$ & $31(17.9)$ & $15(17.1)$ & 0.88 \\
\hline Liver & $38(14.6)$ & $26(15)$ & $12(13.6)$ & 0.78 \\
\hline Brain & $9(3.5)$ & $6(3.5)$ & $3(3.4)$ & 0.73 \\
\hline Pulmonary lymphangitis & $6(2.3)$ & $4(2.3)$ & $2(2.3)$ & 0.67 \\
\hline Missing & $1(0.4)$ & $1(0.6)$ & $0(0)$ & \\
\hline \multicolumn{5}{|l|}{ Anticancer treatment before ICU admission } \\
\hline $\begin{array}{l}\text { Number of previous systemic anti-tumoral treatments } \\
\text { [median (IQR)] }\end{array}$ & $1(0 ; 1)$ & $1(0 ; 1)$ & $1(0 ; 1)$ & 0.32 \\
\hline Missing & $8(3)$ & $5(2.9)$ & $3(3.4)$ & \\
\hline Chemotherapy & $94(56.3)$ & $66(60.6)$ & $28(48.3)$ & 0.18 \\
\hline Targeted therapy & $31(18.7)$ & $22(20.2)$ & $9(15.8)$ & \\
\hline Missing & $95(36.3)$ & $65(37.3)$ & $30(34)$ & \\
\hline \multicolumn{5}{|l|}{ Performance status } \\
\hline 0 or 1 & $98(46.4)$ & $64(45.7)$ & $34(47.9)$ & 0.98 \\
\hline 2 & $86(40.8)$ & $58(41.4)$ & $28(39.4)$ & \\
\hline 3 or 4 & $27(12.9)$ & $18(12.8)$ & $9(12.7)$ & \\
\hline Missing & $51(19.5)$ & 34 (19.5) & $17(19.3)$ & \\
\hline
\end{tabular}

Data are mean \pm SD or number of patients (\%)

ICU admission over the study period $(60.3 \%$ of these had metastatic disease). The presence of a solid tumor did not increase ICU mortality compared with patients who did not have a solid tumor, but elderly patients admitted to the ICU with active cancer were extremely critically ill. The majority of indications for ICU admission were not related to cancer. More than half of patients with an indication for ongoing cancer therapy had treatment resumed after their ICU stay. The 90-day mortality was $51.9 \%$. We identified high SAPS 2 score and primary tumor site as being significantly associated with death at day 90 on multivariate analysis, whereas previous cancer therapies and performance status were not.

In our study, the ICU mortality rate for critically ill elderly patients with solid tumors was $33.6 \%$, comparable to that in similar patients without cancer. Previous 
Table 2 Baseline patient ICU details for the overall study population and by survivor status

\begin{tabular}{|c|c|c|c|c|}
\hline & Overall $(n=262)$ & ICU survivors $(n=174)$ & ICU non-survivors $(n=88)$ & $p$ value \\
\hline Reason for ICU admission [n (\%)] & & & & 0.42 \\
\hline Not related to cancer & $174(66.4)$ & $120(68.9)$ & $54(61.4)$ & \\
\hline Related to anticancer drugs & $49(18.7)$ & $29(16.7)$ & $20(22.7)$ & \\
\hline Related to cancer progression & $39(14.9)$ & $25(14.4)$ & $14(15.9)$ & \\
\hline Reasons for ICU admission [n (\%)] & & & & $<0.001$ \\
\hline Sepsis & $80(30.5)$ & $57(32.8)$ & $3(26.1)$ & \\
\hline Acute respiratory failure & $74(28.2)$ & $47(27)$ & $27(30.8)$ & \\
\hline Coma or seizure & $21(8)$ & $12(6.9)$ & $9(10.2)$ & \\
\hline Cardiorespiratory shock & $18(6.9)$ & $1(0.5)$ & $17(19.3)$ & \\
\hline Hemorrhagic shock & $16(6.1)$ & $14(8.1)$ & $2(2.3)$ & \\
\hline Acute kidney failure & $14(5.3)$ & $10(5.8)$ & $4(4.6)$ & \\
\hline Acute heart failure & $10(3.8)$ & $8(4.6)$ & $2(2.3)$ & \\
\hline Chronic bronchitis exacerbation & $8(3.1)$ & $7(4)$ & $1(1.1)$ & \\
\hline Electrolytic disorders & $6(2.3)$ & $5(2.9)$ & $1(1.1)$ & \\
\hline Hypovolemia & $5(1.9)$ & $4(2.3)$ & $1(1.1)$ & \\
\hline Intentional overdose & $1(0.4)$ & $1(0.5)$ & $0(0)$ & \\
\hline Post-surgery complications & $1(0.4)$ & $1(0.5)$ & $0(0)$ & \\
\hline SAPS 2 score & $61.9 \pm 22.5$ & $51.4 \pm 13.0$ & $82.0 \pm 22.9$ & $<0.001$ \\
\hline Neutropenia & $19(7.3)$ & $12(6.9)$ & $7(8)$ & 0.75 \\
\hline \multicolumn{5}{|l|}{ ICU stay and treatments } \\
\hline Time in ICU [days, median (IQR)] & $4(2 ; 7)$ & $4(2 ; 7) 0$ & $4(1 ; 8)$ & 0.26 \\
\hline Time in hospital [days, median (IQR)] & $14(5 ; 25)$ & $16(8 ; 27)$ & $6(2 ; 16)$ & $<0.001$ \\
\hline Mechanical ventilation & $135(51.5)$ & $54(31)$ & $81(92.1)$ & $<0.0001$ \\
\hline Mechanical ventilation duration (days) & $3.5 \pm 6.3$ & $2.2 \pm 4.4$ & $6.2 \pm 8.3$ & $<0.001$ \\
\hline Noninvasive ventilation & $25(10.3)$ & $20(12.9)$ & $5(5.7)$ & 0.08 \\
\hline Missing & $19(7.3)$ & $19(10.9)$ & $0(0)$ & \\
\hline Inotropes & $126(48.3)$ & $52(30.1)$ & $74(84.1)$ & $<0.0001$ \\
\hline Missing & $1(0.4)$ & $1(0.6)$ & $0(0)$ & \\
\hline Inotropes duration (days) & $1.4 \pm 2.4$ & $0.8 \pm 1.6$ & $2.7 \pm 3.1$ & $<0.001$ \\
\hline Dialysis & $33(12.6)$ & $17(9.8)$ & $16(18.2)$ & 0.05 \\
\hline Missing & $1(0.4)$ & $1(0.6)$ & $0(0)$ & \\
\hline Limitation of active therapies $[n(\%)]$ & $46(17.6)$ & $11(6.3)$ & $35(39.8)$ & $<0.001$ \\
\hline
\end{tabular}

Data are mean \pm SD or number of patients (\%), expect for time in ICU and in hospital (median and interquartile [IQR]). Time in ICU and in hospital is median (IQR)

studies assessing the outcome of cancer patients in the ICU reported a wide range of mortality rates, from 24 to $75 \%[9,11-15]$. This wide range can be explained by the marked heterogeneity in patient case mix: medical and surgical patients; solid and hematological cancer patients; allogeneic and autologous bone marrow transplant recipients; and patients with and without early limitation of active therapies $[5,16]$. In many studies, hematological cancer, medical admissions and limitation of active therapies were associated with worse prognosis [16]. In our study, we focused on patients with solid cancers and we excluded those with early limitation of active therapies.

To date, few studies in this setting have focused on elderly patients. In a registry of 1134 patients aged $>65$ years with advanced lung cancer admitted to ICUs, the in-hospital mortality was 33\% [8], almost identical to the rate in our study. In the registry cohort, age was not associated with mortality, and this was also the case in multivariate analyses from other studies assessing predictors of death in critically ill patients admitting to the ICU with solid cancers [11, 12, 15-17].

In our cohort, patient prognosis after ICU admission appeared to be associated more with the severity of clinical condition at ICU admission than with the presence of cancer and age, as in most other previous studies $[8,9$, 11-17]. In the Sepsis Occurrence in Acutely Ill Patients (SOAP) study, patients with solid tumors had similar survival to patients without cancer, as was the case in our analysis, although the SOAP study included patients aged $<65$ years [18]. Also in the SOAP study, hospital mortality 
Table 3 Independent predictors of 90-day mortality in elderly solid tumor patients $(n=262)$

\begin{tabular}{|c|c|c|c|c|}
\hline & \multicolumn{2}{|l|}{ Univariate } & \multicolumn{2}{|l|}{ Multivariate } \\
\hline & $\mathrm{HR}(95 \% \mathrm{Cl})$ & $p$ value & $\mathrm{HR}(95 \% \mathrm{Cl})$ & $p$ value \\
\hline Age (HR for increase of 1 year) & $0.98(0.95-1.01)$ & 0.16 & $0.99(0.95-1.03)$ & 0.57 \\
\hline \multicolumn{5}{|l|}{ Sex } \\
\hline Male & 1 & & 1 & \\
\hline Female & $0.66(0.46-0.96)$ & 0.03 & $0.75(0.43-1.30)$ & 0.30 \\
\hline \multicolumn{5}{|l|}{ Performance status } \\
\hline $0-1$ & 1 & 0.07 & 1 & 0.17 \\
\hline 2 & $1.30(0.85-1.97)$ & & $1.58(0.97-2.59)$ & \\
\hline $3-4$ & $1.88(1.10-3.23)$ & & $1.53(0.77-3.05)$ & \\
\hline \multicolumn{5}{|l|}{ Primary tumor site } \\
\hline Genitourinary & 1 & 0.005 & 1 & 0.01 \\
\hline Gastrointestinal & $1.10(0.65-1.86)$ & & $0.96(0.49-1.89)$ & \\
\hline Breast & $0.82(0.36-1.89)$ & & $0.38(0.11-1.27)$ & \\
\hline Lung & $1.37(0.83-2.26)$ & & $1.11(0.59-2.08)$ & \\
\hline Head and neck & $2.60(1.49-4.53)$ & & $2.69(1.25-5.79)$ & \\
\hline Other & $0.75(0.29-1.96)$ & & $0.80(0.24-2.67)$ & \\
\hline \multicolumn{5}{|l|}{ Metastatic status } \\
\hline No & 1 & & 1 & \\
\hline Yes & $0.96(0.68-1.36)$ & 0.82 & $1.61(0.98-2.59)$ & 0.06 \\
\hline Number of previous systemic anti-tumoral treatments & $0.88(0.73-1.07)$ & 0.19 & $(-)$ & \\
\hline \multicolumn{5}{|l|}{ Reason for ICU admission } \\
\hline Not related to cancer & $1(-)$ & 0.68 & 1 & 0.50 \\
\hline Related to anticancer drugs & $1.07(0.69-1.67)$ & & $0.87(0.49-1.52)$ & \\
\hline Related to cancer progression & $1.23(0.76-2.00)$ & & $1.23(0.66-2.28)$ & \\
\hline SAPS 2 score (HR for increase of 1 unit) & $1.05(1.04-1.06)$ & $<0.0001$ & $1.05(1.03-1.06)$ & $<0.0001$ \\
\hline \multicolumn{5}{|l|}{ Life-support techniques } \\
\hline Mechanical ventilation use & $5.96(3.91-9.10)$ & $<0.0001$ & $(-)$ & \\
\hline Noninvasive ventilation use & $0.81(0.46-1.44)$ & 0.48 & $(-)$ & \\
\hline Inotropes use & $3.68(2.54-5.33)$ & $<0.0001$ & $(-)$ & \\
\hline Dialysis use & $1.28(0.79-2.08)$ & 0.32 & $(-)$ & \\
\hline Limitation of active therapies during ICU stay & $3.79(2.59-5.56)$ & $<0.0001$ & $(-)$ & \\
\hline \multicolumn{5}{|l|}{ Laboratory findings (HR for increase of 1 unit) } \\
\hline Albumin & $0.94(0.89-0.99)$ & 0.02 & $(-)$ & \\
\hline Leukocytes & $1.01(1.01-1.02)$ & $<0.0001$ & $0.99(0.97-1.01)$ & 0.57 \\
\hline Glycemia & $1.03(1.00-1.05)$ & 0.04 & $1.01(0.98-1.06)$ & 0.46 \\
\hline $\mathrm{pH}$ & $0.04(0.01-0.13)$ & $<0.0001$ & $0.85(0.16-4.41)$ & 0.84 \\
\hline Lactates & $1.18(1.13-1.22)$ & $<0.0001$ & $1.07(0.99-1.15)$ & 0.08 \\
\hline ASAT & $1(1-1.001)$ & $<0.0001$ & $1(1-1)$ & 0.58 \\
\hline
\end{tabular}

ASAT aspartate aminotransferase, $\mathrm{Cl}$ confidence interval, $H R$ hazard ratio

increased as the number of organs failing increased, and more than $75 \%$ of the subset of patients with more than three failing organs died compared with about $50 \%$ of patients without cancer $(p=0.01)$. In a multicenter study conducted in Brazil $(n=717)$, higher Sequential Organ Failure Assessment scores were found to be associated with increased hospital mortality on multivariate analysis (odds ratio [OR] 1.25; 95\% confidence interval [CI] 1.171.34) [11].
We found that primary cancer type was associated with 90-day mortality whereas number of previous cancer treatments was not. This is an area of debate, with recent studies that assessed factors associated with ICU death in cancer patients regardless of age and cancer type showing an inconsistent link between ICU death and cancer $[5,9,16,19,20]$; some authors have found association between cancer progression, metastatic status and prognosis $[9,11,15]$. In a large multicenter study including 717 
Table 4 Multivariate analysis of factors associated with definitive anticancer drug cessation after ICU discharge in survivors

\begin{tabular}{|c|c|c|c|}
\hline & OR & $95 \% \mathrm{Cl}$ & $p$ value \\
\hline \multicolumn{4}{|l|}{ Primary tumor site } \\
\hline Genitourinary & 1 & & \\
\hline Gastrointestinal & 8.70 & $1.61-47.15$ & 0.01 \\
\hline Lung & 6.92 & $1.28-37.38$ & 0.02 \\
\hline Breast & 1.41 & $0.10-20.17$ & 0.80 \\
\hline Other & 5.50 & $0.71-42.68$ & 0.10 \\
\hline \multicolumn{4}{|l|}{ Performance status } \\
\hline 0 or 1 & 1 & & \\
\hline 2 & 2.26 & $0.82-7.60$ & 0.14 \\
\hline 3 or 4 & 14.96 & $2.71-78.68$ & 0.001 \\
\hline \multicolumn{4}{|l|}{ Reason for ICU admission } \\
\hline Not related to cancer & 1 & & \\
\hline Related to anticancer drugs & 1.10 & $0.76-6.69$ & 0.87 \\
\hline Related to cancer progression & 4.19 & $1.03-17.14$ & 0.04 \\
\hline \multicolumn{4}{|l|}{ Other } \\
\hline LAT in ICU & $191,146,845.57$ & $0-\operatorname{lnf}$ & 0.99 \\
\hline Dialysis & 3.19 & $0.67-15.28$ & 0.14 \\
\hline High blood bilirubin level & 1.00 & $0.99-1.02$ & 0.48 \\
\hline
\end{tabular}

$\mathrm{Cl}$ confidence interval, $O R$ odds ratio, $L A T$ limitation of active therapies

patients (667 with solid tumors and 50 with hematological malignancies), active recurrent or progressive malignancy was associated with increased hospital mortality in multivariate analysis [11, 17]. Another study among patients with solid tumors $(n=162)$ found that complete or partial remission of the underlying cancer decreased the risk of ICU mortality (OR $0.026,95 \%$ CI $0.002-0.3$; $p=0.004$ ) [15]. Our data showed that ICU admission related to tumor progression was not associated with mortality, but was associated with less resumption of anticancer treatment. However, we did exclude patients with early limitation of active therapies, and most admissions were not related to cancer progression. It has previously been shown that some specific causes of admission due to progression of solid tumors are associated with very poor prognosis $[21,22]$. As a result, the elderly patients admitted to the ICU in this study might represent a selected group, and we did not assess patients not referred to the ICU by the oncologist or refused by the intensivist.

There is a relative lack of data about links between long-term outcome and anticancer therapy resumption $[8,9]$. In our study, the number of patients able to resume antineoplastic therapy was encouraging (52.7\%). This rate is high compared to the small amount of existing data. In one study of 1134 patients aged $>65$ years with active advanced lung cancer, only 19\% resumed anticancer therapy after ICU discharge [8]. Another study about critically ill lung cancer patients reported a therapy resumption rate of $37 \%$ [9]. Possible reasons for the lower rates in the other studies could be the advanced stage of lung cancer, the high proportion of cancer-related complications and low number of therapeutic options in those patients. In addition, new targeted therapies in oncology and the fact that our cohort included other tumor types could have contributed to the different results between studies. It has been previously reported that there is a link between poor performance status and no resumption of anticancer treatment after an ICU stay [9]. Similarly, we found that gastrointestinal and lung cancer or patient admitted to the ICU because of tumor progression, or with poor performance status were prognostic factors for no anticancer therapy after ICU discharge. One explanation for this could be that patients with tumor progression may be more weakened by an ICU stay, and thus, further cancer therapy might be deemed too intense for them.

This study had several strengths. It is one of the largest cohorts in which long-term mortality of elderly patients with active cancer admitted to the ICU has been determined. Moreover, only patients with solid tumors were included, eliminating high-risk populations (e.g., patients with hematological malignancies. Another strength was the collection of data about tumor and metastatic sites, as well as the number and type of treatments received prior to ICU admission. Furthermore, we include data on resumption of anticancer therapy after ICU discharge, something that has only been reported in a few previous studies and is clinically relevant because it could play a part in assessing whether or not to admit a patient with cancer to the ICU.

Our study also has some limitations that need to be taken into account when interpreting the data. The study was conducted in a single center and had a retrospective design. Also, there were some database limitations, including missing data on biological and clinical variables (e.g., albumin level or performance status) meaning that calculations on the prognostic value of these variables may be affected. Furthermore, our results are only applicable to a subset of similar patients due to selection bias; patients too unwell to be admitted to the ICU were not included, as indicated by the fact that only $15 \%$ of our patients were in the ICU because of cancer progression. As a result, the study population had a relatively better general condition with fewer comorbidities, which may explain the good outcomes observed.

\section{Conclusions}

Our study results showed that elderly patients ( $\geq 65$ years) with solid tumor admitted to the ICU had the same mortality rate as similar elderly patients without 
cancer. In addition, more than half of the elderly cancer patients discharged from the ICU were able to resume anticancer treatment. Factors associated with ICU mortality were related to the patients' status at ICU admission and not to the presence of metastatic disease or the number of previous anticancer treatments. These findings suggest that early admission of elderly cancer patients to the ICU is appropriate, when necessary.

\section{Additional file}

Additional file 1: Table S1. Main characteristics of the whole population. Table S2. Biological data at admission in cancer patients $(n=262)$. Table S3. Characteristics of ICU survivors with anti tumoral treatment indication according to cessation/resumption of anti cancer drugs after ICU discharge. Table S4. Independent predictors of 90-days mortality (multivariate analysis including life supporting therapies).

\section{Abbreviations}

95\% Cl: 95\% confidence interval; HR: hazard ratio; ICU: intensive care unit; LATA: limitation of active therapies; SOAP: Sepsis Occurrence in Acutely III Patients.

\section{Authors' contributions}

EA contributed to protocol elaboration, data collection, statistics, manuscript writing. AC-N and SK participated in statistics, manuscript reviewing. BA collected data. EG, SO, CH-B, CT, AM, J-LD, J-YF and JT involved in protocol elaboration, manuscript reviewing. NA participated in protocol elaboration, manuscript writing, manuscript reviewing, coordination of the study. All authors read and approved the final manuscript.

\section{Author details}

${ }^{1}$ Gastrointestinal Oncology Department, European Georges Pompidou Hospital, Paris, France. ${ }^{2}$ Intensive Care Unit, European Georges Pompidou Hospital, Paris, France. ${ }^{3}$ Oncology Department, European Georges Pompidou Hospital, Paris, France. ${ }^{4}$ Université Paris Descartes, Paris, France. ${ }^{5}$ Clinical Research Unit, European Georges Pompidou Hospital, Paris, France.

\section{Acknowledgements}

English language editing assistance was provided by Nicola Ryan, independent medical writer.

\section{Competing interests}

The authors declare that they have no competing interests.

\section{Availability of data and materials}

The dataset supporting the conclusions of this article is available by contacting the corresponding author.

\section{Ethics approval and consent to participate}

The protocol was approved by the local institutional ethics committee, and the study was performed in accordance with the ethical standards laid down in the 1964 Declaration of Helsinki.

Received: 11 August 2016 Accepted: 23 February 2017

Published online: 06 March 2017

\section{References}

1. Institut National du Cancer. Les cancers en France en 2015, l'essentiel des faits et chiffres [Internet]. http://www.e-cancer.fr/Expertises-et-publications/Catalogue-des-publications/Les-cancers-en-France-en-2015-Lessentiel-des-faits-et-chiffres; 2016.
2. Annual Report to the Nation [Internet]. National Cancer Institute. [cited 2016 Jan 19]. http://www.cancer.gov/research/progress/ annual-report-nation.

3. Staudinger T, Stoiser B, Müllner M, Locker GJ, Laczika K, Knapp S, et al. Outcome and prognostic factors in critically ill cancer patients admitted to the intensive care unit. Crit Care Med. 2000;28(5):1322-8.

4. Ferlay J, Steliarova-Foucher E, Lortet-Tieulent J, Rosso S, Coebergh JWW, Comber H, et al. Cancer incidence and mortality patterns in Europe: estimates for 40 countries in 2012. Eur J Cancer Oxf Engl 1990. 2013;49(6):1374-403.

5. Azoulay E, Soares M, Darmon M, Benoit D, Pastores S, Afessa B. Intensive care of the cancer patient: recent achievements and remaining challenges. Ann Intensive Care. 2011;1 (1):5.

6. Reichner CA, Thompson JA, O'Brien S, Kuru T, Anderson ED. Outcome and code status of lung cancer patients admitted to the medical ICU. Chest. 2006;130(3):719-23.

7. Roques S, Parrot A, Lavole A, Ancel P-Y, Gounant V, Djibre M, et al. Sixmonth prognosis of patients with lung cancer admitted to the intensive care unit. Intensive Care Med. 2009;35(12):2044-50.

8. Bonomi MR, Smith CB, Mhango G, Wisnivesky JP. Outcomes of elderly patients with stage IIIB-IV non-small cell lung cancer admitted to the intensive care unit. Lung Cancer Amst Neth. 2012;77(3):600-4.

9. Soares M, Toffart A-C, Timsit J-F, Burghi G, Irrazábal C, Pattison N, et al. Intensive care in patients with lung cancer: a multinational study. Ann Oncol Off J Eur Soc Med Oncol ESMO. 2014;25(9):1829-35.

10. Le Gall JR, Lemeshow S, Saulnier F. A new Simplified Acute Physiology Score (SAPS II) based on a European/North American multicenter study. JAMA. 1993;270(24):2957-63.

11. Soares M, Caruso P, Silva E, Teles JMM, Lobo SMA, Friedman G, et al. Characteristics and outcomes of patients with cancer requiring admission to intensive care units: a prospective multicenter study. Crit Care Med. 2010;38(1):9-15.

12. Slatore CG, Cecere LM, Letourneau JL, O'Neil ME, Duckart JP, Wiener RS, et al. Intensive care unit outcomes among patients with lung cancer in the surveillance, epidemiology, and end results-medicare registry. J Clin Oncol Off J Am Soc Clin Oncol. 2012;30(14):1686-91.

13. Azoulay E, Alberti C, Bornstain C, Leleu G, Moreau D, Recher C, et al. Improved survival in cancer patients requiring mechanical ventilatory support: impact of noninvasive mechanical ventilatory support. Crit Care Med. 2001;29(3):519-25.

14. Puxty K, McLoone P, Quasim T, Sloan B, Kinsella J, Morrison DS. Risk of critical illness among patients with solid cancers: a population-based observational study. JAMA Oncol. 2015;1(8):1078-85.

15. Aygencel G, Turkoglu M, Turkoz Sucak G, Benekli M. Prognostic factors in critically ill cancer patients admitted to the intensive care unit. J Crit Care. 2014;29(4):618-26.

16. Taccone FS, Artigas AA, Sprung CL, Moreno R, Sakr Y, Vincent J-L. Characteristics and outcomes of cancer patients in European ICUs. Crit Care Lond Engl. 2009;13(1):R15.

17. Azevedo LCP, Caruso P, Silva UVA, Torelly AP, Silva E, Rezende E, et al. Outcomes for patients with cancer admitted to the ICU requiring ventilatory support: results from a prospective multicenter study. Chest. 2014;146(2):257-66.

18. Sprung CL, Sakr Y, Vincent J-L, Le Gall J-R, Reinhart K, Ranieri VM, et al. An evaluation of systemic inflammatory response syndrome signs in the Sepsis Occurrence In Acutely III Patients (SOAP) study. Intensive Care Med. 2006;32(3):421-7.

19. Puxty K, McLoone P, Quasim T, Kinsella J, Morrison D. Survival in solid cancer patients following intensive care unit admission. Intensive Care Med. 2014;40(10):1409-28.

20. Toffart A-C, Minet C, Raynard B, Schwebel C, Hamidfar-Roy R, Diab S, et al. Use of intensive care in patients with nonresectable lung cancer. Chest. 2011;139(1):101-8.

21. Meert A-P, Berghmans T, Hardy M, Markiewicz E, Sculier J-P. Non-invasive ventilation for cancer patients with life-support techniques limitation. Support Care Cancer Off J Multinatl Assoc Support Care Cancer. 2006;14(2):167-71.

22. Oh SY, Lee S-J, Lee J, Lee S, Kim S-H, Kwon H-C, et al. Gastric leptomeningeal carcinomatosis: multi-center retrospective analysis of 54 cases. World J Gastroenterol. 2009;15(40):5086-90. 